\title{
The role of spatial attention in the processing of facial expression: An ERP study of rapid brain responses to six basic emotions
}

\author{
MARTIN EIMER and AMANDA HOLMES \\ University of London, London, England \\ and \\ FRANCIS P. MCGLONE \\ Unilever Research, Port Sunlight Laboratories, Wirral, England
}

\begin{abstract}
To investigate the time course of emotional expression processing, we recorded ERP responses to stimulus arrays containing neutral versus angry, disgusted, fearful, happy, sad, or surprised faces. In one half of the experiment, the task was to discriminate emotional and neutral facial expressions. Here, an enhanced early frontocentral positivity was elicited in response to emotional as opposed to neutral faces, followed by a broadly distributed positivity and an enhanced negativity at lateral posterior sites. These emotional expression effects were very similar for all six basic emotional expressions. In the other half of the experiment, attention was directed away from the faces toward a demanding perceptual discrimination task. Under these conditions, emotional expression effects were completely eliminated, demonstrating that brain processes involved in the detection and analysis of facial expression require focal attention. The face-specific N170 component was unaffected by any emotional expression, supporting the hypothesis that structural encoding and expression analysis are independent processes.
\end{abstract}

Emotions play a crucial role in the regulation of interactions between humans and their environment. Emotional states produce specific bodily responses, aimed at preparing the organism for survival-related behavior, and specialized neural systems have evolved for the rapid perceptual analysis of emotionally salient external events, such as emotional facial expressions (Damasio, 1994; Le Doux, 1996; Öhman, Flykt, \& Lundqvist, 2000). Attentional biases toward emotional stimuli have been found in many behavioral studies, using paradigms such as visual search (Eastwood, Smilek, \& Merikle, 2001; Fox et al., 2000; Hansen \& Hansen, 1988; Öhman, Flykt, \& Esteves, 2001; Öhman, Lundqvist, \& Esteves, 2001) and dot probe detection tasks (Mogg \& Bradley, 1999; Mogg et al., 2000).

Numerous studies have demonstrated an important role of the amygdala in detecting emotionally salient events and in mediating responses to these stimuli. Emotional stimuli, particularly fearful facial expressions, activate the amygdala and other connected limbic structures (Liu, Ioannides, \& Streit, 1999; Morris et al., 1996). Reentrant projections from the amygdala back to occipital cortex may be involved in an enhancement of visual processing of emotionally salient stimuli (Amaral \& Price, 1984; Amaral, Price, Pitkanen, \& Carmichael,

This study was supported by Unilever Research. We thank two anonymous referees for valuable comments, and Heijo Van de Werf for technical assistance. Correspondence should be addressed to M. Eimer, Department of Psychology, Birkbeck College, University of London, Malet Street, London WC1E 7HX, England (e-mail: m.eimer@bbk.ac.uk).
1992; see also Armony \& Dolan, 2002; Lang et al., 1998; Morris et al., 1998, for supportive evidence from functional imaging studies).

Given these findings, it is often assumed that emotional stimuli are detected preattentively and then automatically trigger attentional shifts toward their location. However, recent studies investigating the relationship between spatial attention and the processing of emotionally salient events have yielded conflicting findings. On the one hand, amygdala responses to fearful faces in humans appear to be unaffected by spatial attention (Vuilleumier, Armony, Driver, \& Dolan, 2001), and amygdala activations triggered by highly arousing emotional scenes are not modulated by a secondary task (Lane, Chua, \& Dolan, 1999). In addition, neglect and extinction patients are more likely to detect emotionally significant relative to neutral pictures when these are presented in the affected visual hemifield (Vuilleumier \& Schwartz, 2001a, 2001b). These results suggest that emotional stimuli capture attention automatically. On the other hand, amygdala responses to fearful or happy facial expressions have been found to be modulated by focal attention (Pessoa, McKenna, Gutierrez, \& Ungerleider, 2002), and increased responses to attended versus unattended fearful faces have been observed in the anterior temporal pole and anterior cingulate gyrus (Vuilleumier et al., 2001).

In the present study, we used event-related brain potential (ERP) measures to further investigate the role of spatial attention in the processing of emotionally significant events. Because of their excellent temporal resolution, 
ERPs are particularly suited for studying the time course of emotional processes and investigating whether and when the processing of emotional stimuli is modulated by selective attention. For example, a positive slow wave starting at about $300 \mathrm{msec}$ after stimulus onset in response to pictures with emotional content (Cuthbert, Schupp, Bradley, Birbaumer, \& Lang, 2000; Diedrich, Naumann, Maier, \& Becker, 1997) has been interpreted as reflecting the allocation of attention to motivationally relevant input (Cuthbert et al., 2000). More recently, we have shown that an enhanced positivity in response to foveally presented fearful relative to neutral faces can be elicited over prefrontal areas as early as $120 \mathrm{msec}$ after stimulus onset (Eimer \& Holmes, 2002). This early emotional expression effect suggests that cortical circuits involved in the detection of emotionally significant events can be triggered rapidly by emotional facial expressions (see also Kawasaki et al., 2001; Pizzagalli, Regard, \& Lehmann, 1999; Sato, Kochiyama, Yoshikawa, \& Matsumura, 2001, for similar results from ERP and single-unit studies).

In another recent ERP study (Holmes, Vuilleumier, \& Eimer, 2003), we investigated for the first time whether and how emotional expression effects elicited by fearful relative to neutral faces are affected by spatial attention. On each trial, arrays consisting of two faces and two houses arranged in horizontal and vertical pairs were presented. Participants had to attend either to the two vertical or to the two horizontal locations (as indicated by a precue presented at the beginning of each trial) in order to detect infrequent identical stimuli at the cued location. When faces were attended, fearful faces elicited an enhanced positivity relative to neutral faces, with an early frontal effect followed by a more broadly distributed emotional positivity. These emotional expression effects were completely eliminated on trials where faces were presented at uncued (unattended) locations. This finding challenges the hypothesis that the detection and processing of emotional facial expression occurs preattentively and suggests that the processes reflected by ERP modulations sensitive to emotional facial expression are gated by spatial attention.

The present study was designed to confirm and extend these surprising results. In our previous study (Holmes et al., 2003), only one emotional facial expression (fear) was employed. Although fearful faces are generally regarded to be highly salient emotional stimuli, the hypothesis that the processing of emotional facial expression depends on spatial attention clearly needs to be substantiated by investigating whether differential ERP responses to facial expressions other than fear are gated by spatial attention.

In the present experiment, all six basic emotional facial expressions were shown in separate experimental blocks. Face stimuli were photographs of 10 different individuals (Ekman \& Friesen, 1976), with facial expression neutral or angry, disgusted, fearful, happy, sad, or surprised (Figure 1, top panel). Each block contained an equal number of trials with emotional or neutral face pairs presented bilaterally to the left and right of fixation. In one half of the experiment (lines task), attention was actively directed away from these face stimuli toward a demanding perceptual judgment task. Participants had to monitor a pair of vertical lines presented bilaterally close to fixation (Figure 1, bottom panel) in order to decide on each trial whether the two lines were identical or differed in length. Faces had to be entirely ignored. The other half of the experiment (emotion task) was physically identical to the lines task, but participants now had to decide on each trial whether facial ex pression was emotional or neutral. Here, lines could be entirely ignored.

ERP modulations sensitive to emotional facial expression were identified by comparing ERPs elicited by arrays containing emotional faces to ERPs in response to arrays with neutral faces, separately for experimental blocks including angry, disgusted, fearful, happy, sad, and surprised faces. To investigate the impact of attention on the processing of emotional facial expression, these comparisons were conducted separately for the emotion task, where emotional expression was task relevant, and for the lines task, where faces were irrelevant and thus could be entirely ignored. If emotional facial expressions were detected preattentively and attracted attention automatically, systematic ERP modulations in response to arrays containing emotional versus neutral faces should be found not only in the emotion task, but also, although perhaps in an attenuated fashion, in the lines task. In contrast, if the detection and processing of emotional faces requires focal attention (as suggested by Holmes et al., 2003), ERP correlates of emotional face processing should be entirely absent in the lines task.

In addition to investigating the role of spatial attention on the processing of facial expression, the design of the present study also allowed the systematic comparison of ERP responses elicited by each of the six basic emotional facial expressions. A number of lesion and neuroimaging studies argue for the existence of neural systems that are specialized for processing distinct emotions (Adolphs, 2002). For example, a disproportionate activation of the amygdala has been observed in response to facial expressions of fear (Breiter et al., 1996; Morris et al., 1996; Phillips et al., 1998; Whalen et al., 2001; but see Rapcsak et al., 2000). Prefrontal cortex has been specifically implicated in the recognition of angry facial expressions (Blair, Morris, Frith, Perrett, \& Dolan, 1999; Harmer, Thilo, Rothwell, \& Goodwin, 2001), and the insula and basal ganglia appear to be particularly involved in processing facial expressions of disgust (Adolphs, Tranel, \& Damasio, 2003; Calder, Keane, Manes, Antoun, \& Young, 2000; Calder, Lawrence, \& Young, 2001; Phillips et al., 1998; Phillips et al., 1997; Sprengelmeyer, Rausch, Eysel, \& Przuntek, 1998). If the detection and analysis of specific facial emotional expressions is mediated by distinct brain processes, this might be reflected in systematic differences in emotional expression effects on ERP waveforms elicited in response to different facial expressions.

Another aim in the present study was to investigate whether early stages in the perceptual encoding of face stimuli are affected by emotional facial expression. The 

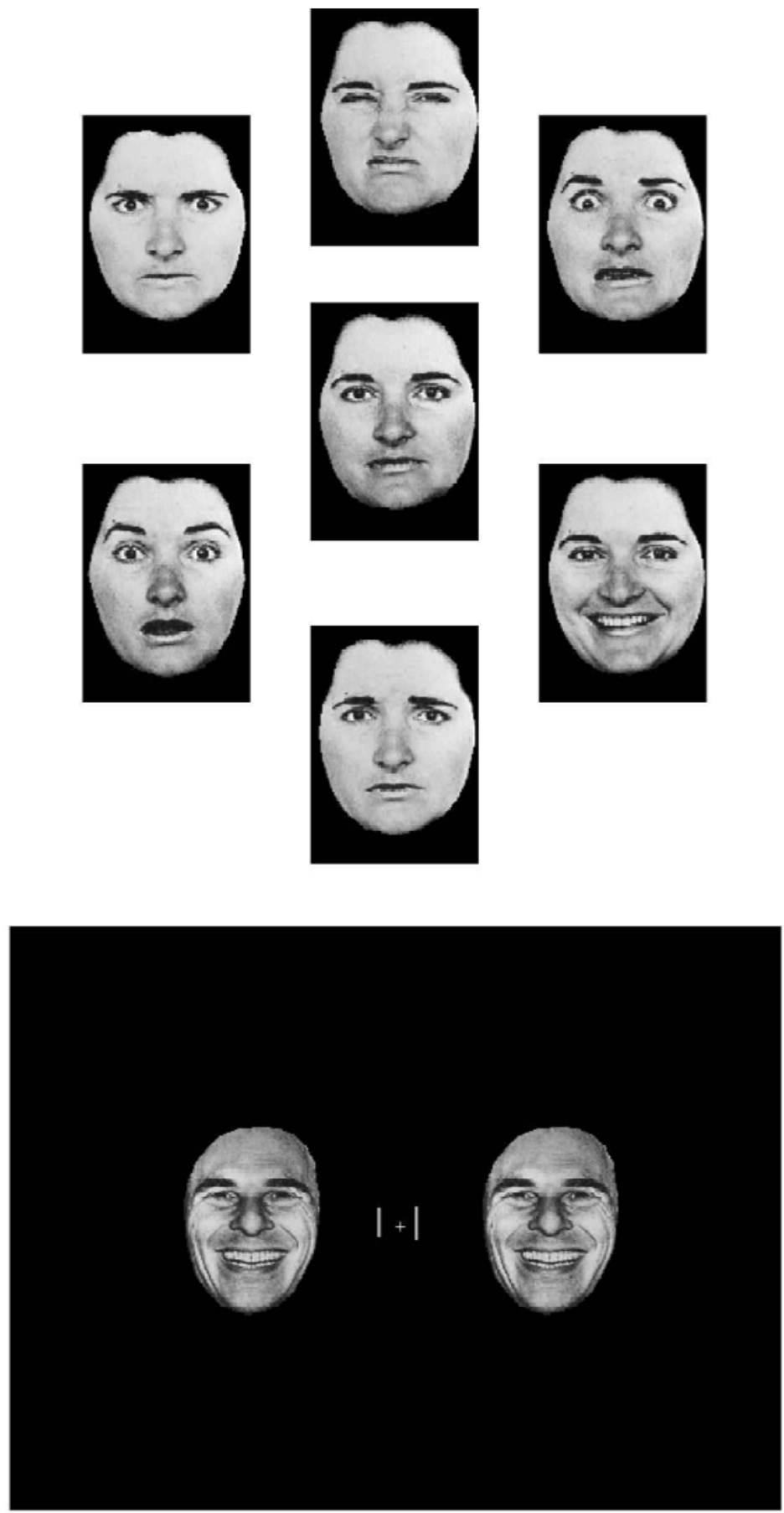

Figure 1. Top panel: Examples of face stimuli used in the present experiment. Faces of 10 different individuals were used, with facial expression either neutral (central), or (clockwise from top) disgusted, fearful, happy, sad, surprised, or angry. Bottom panel: Illustration of the stimulus array presented on each trial. Two identical emotional or neutral faces were presented bilaterally with two vertical lines located close to fixation. In the trial shown here, a happy face pair is presented together with two lines of different lengths.

face-specific N170 component is assumed to reflect the precategorical structural encoding of faces prior to their recognition (Bentin, Allison, Puce, Perez, \& McCarthy, 1996; Eimer, 1998, 2000). In two recent ERP studies
(Eimer \& Holmes, 2002; Holmes et al., 2003), we have found that the $\mathrm{N} 170$ is not modulated by emotional facial expression. This suggests that the structural encoding of faces and the processing of emotional expression are 
parallel and independent processes (Bruce \& Young, 1986). However, to date this conclusion has been based only on a comparison of N170 components elicited in response to fearful as versus neutral faces, obtained under conditions where facial expression was task irrelevant. To investigate whether the face-specific N170 is unaffected by any emotional facial expression, even when expression is task relevant, we compared the N170 elicited by emotional versus neutral faces in the emotion task, separately for all six basic facial expressions. Any systematic emotional expression effects on the N170 component would challenge the hypothesis that the structural encoding of faces is completely independent of facial expression analysis.

\section{METHOD}

\section{Participants}

Fifteen participants participated in this study. One had to be excluded because of excessive eye blinks, so 14 participants ( 7 female and 7 male; 18-54 years old; average age, 29.6 years) remained in the sample. One participant was left-handed, all others right-handed, by self-report. The experiment was performed in compliance with relevant institutional guidelines and was approved by the Birkbeck College School of Psychology ethics committee.

\section{Stimuli}

The face stimuli were photographs of faces of 10 different individuals, all taken from a standard set of pictures of facial affect (Ekman \& Friesen, 1976). Facial expression was angry, disgusted, fearful, happy, sad, surprised, or neutral, resulting in a total of 70 different face stimuli (see Figure 1, top panel, for examples). All face stimuli covered a visual angle of about $3.4^{\circ} \times 2.4^{\circ}$. Each display also contained a pair of gray vertical lines $\left(0.1^{\circ}\right.$ width $)$, and each line was either short $\left(0.4^{\circ}\right)$ or slightly longer $\left(0.5^{\circ}\right)$. All stimuli were presented on a computer screen in front of a black background. A white fixation cross was continuously present at the center of the screen.

\section{Procedure}

Participants were seated in a dimly lit sound-attenuated cabin, and a computer screen was placed at a viewing distance of $70 \mathrm{~cm}$. The experiment consisted of 24 experimental blocks, each containing 80 trials. On each trial, two identical faces were presented together with two line stimuli in front of a black background (Figure 1 , bottom). Faces were located $2.2^{\circ}$ to the left and right of fixation (measured as the distance between the fixation cross and the center of each face stimulus), and the bilateral lines were presented close to the fixation cross $\left(0.4^{\circ}\right.$ eccentricity). All stimuli were presented simultaneously for $300 \mathrm{msec}$, and the interval between two successive stimulus presentations was $2,000 \mathrm{msec}$.

In 12 successive blocks, participants had to indicate with a lefthand or right-hand buttonpress whether the face pair presented on any given trial showed an emotional or neutral expression (emotion task). The mapping of emotional valence to response hand was counterbalanced across participants. In 40 trials per block, emotional faces were presented; in the other randomly intermingled 40 trials, facial expression was neutral. Long and short lines, which were irrelevant in these blocks, appeared randomly and with equal probability to the left and right of fixation. Emotional expression was varied across blocks, with angry, disgusted, fearful, happy, sad, and surprised faces each shown in two blocks. The order in which these blocks were presented was randomized for each participant.

In the other 12 successive blocks, participants were instructed to direct their attention to the pair of lines presented close to fixation and to indicate with a left-hand or right-hand buttonpress whether these lines differed in length or were identical (lines task). The mapping of line length to response hand was counterbalanced across participants. Again, short and long lines appeared randomly and equiprobably on the left or right side. Faces, which were now task irrelevant, were emotional on 40 trials and neutral on the other 40 trials, with emotional expression varied across blocks (two blocks each with angry, disgusted, fearful, happy, sad, and surprised faces). The order in which these blocks were presented was again randomized for each participant.

Seven participants performed the emotion task prior to the lines task, and this order was reversed for the other 7 participants. Participants were instructed to keep their gaze directed at the central fixation cross throughout each block and to respond as fast and accurately as possible on each trial.

\section{ERP procedures and data analysis}

EEG was recorded with $\mathrm{Ag}-\mathrm{AgCl}$ electrodes and linked-earlobe reference from Fpz, F7, F3, Fz, F4, F8, FC5, FC6, T7, C3, Cz, C4, T8, CP5, CP6, T5, P3, Pz, P4, T6, and Oz (according to the 10-20 system), and from OL and OR (located halfway between O1 and P7, and $\mathrm{O} 2$ and $\mathrm{P} 8$, respectively). Horizontal EOG(HEOG) was recorded bipolarly from the outer canthi of both eyes. The impedance for all electrodes was kept below $5 \mathrm{k} \Omega$. The amplifier bandpass was 0.1 to $40 \mathrm{~Hz}$, and no additional filters were applied to the averaged data. EEG and EOG were sampled with a digitization rate of $200 \mathrm{~Hz}$ and stored on disk. Reaction times (RTs) were measured on each trial.

EEG and HEOG were epoched off-line into 800 -msec periods, starting $100 \mathrm{msec}$ prior to stimulus onset and ending $700 \mathrm{msec}$ after stimulus onset. Trials with horizontal eye movements (HEOG exceeding $\pm 30 \mu \mathrm{V}$ ), eyeblinks (Fpz exceeding $\pm 60 \mu \mathrm{V}$ ), or other artifacts (a voltage exceeding $\pm 80 \mu \mathrm{V}$ at any electrode) measured after stimulus onset were excluded from analysis. EEG obtained was averaged relative to a $100-\mathrm{msec}$ baseline preceding stimulus onset. Only trials with correct behavioral responses were included in the averages. Separate averages were computed for the emotion task and the lines task, for all combinations of block type (experimental blocks including angry vs. disgusted vs. fearful vs. happy vs. sad vs. surprised faces) and valence (emotional vs. neutral faces), resulting in 24 average waveforms for each electrode and participant.

The first set of analyses was based on mean amplitudes obtained at lateral posterior electrodes T5 and T6 (where the N170 is maximal) within a time window centered on the mean latency of the facespecific posterior N170 component (160-200 msec poststimulus). Repeated measures analyses of variance (ANOVAs) were conducted for the factors task (emotion task vs. lines task), block type, and valence. Additional analyses were conducted separately for the emotion and the lines tasks. The second set of analyses was based on mean amplitude values computed within five successive poststimulus time windows (120-155 msec, 160-215 msec, 220-315 msec, $320-495 \mathrm{msec}$, and 500-700 msec), which covered the interval where systematic emotional expression effects were observed in our previous experiments (Eimer \& Holmes, 2002; Holmes et al., 2003). Mean amplitude values were computed for frontal (F3, Fz, F4), central $(\mathrm{C} 3, \mathrm{Cz}, \mathrm{C} 4)$, parietal $(\mathrm{P} 3, \mathrm{Pz}, \mathrm{P} 4)$, lateral temporal (T5, T6), and lateral occipital sites (OL, OR). Again, ANOVAs were conducted for the factors task, block type, and valence, followed by further analyses conducted separately for ERPs obtained in the emotion task and the lines task.

For keypress responses, repeated measures ANOVAs were performed on the latencies of correct responses and on error rates, separately for the emotion task and the lines task, for the factors block type and valence. In the analysis of behavioral performance in the lines task, the additional factor of target type (identical lines vs. different lines) was included. For all analyses, Greenhouse-Geisser adjustments to the degrees of freedom were performed when appropriate. 


\section{RESULTS}

\section{Behavioral Results}

Participants failed to respond on less than $3 \%$ of all trials. Correct responses were faster in the emotion task $(622 \mathrm{msec})$ than in the lines task $(695 \mathrm{msec})$, and this difference was significant $[t(14)=4.74, p<.001]$. Figure 2 shows mean RTs (top panel) and the percentage of incorrect responses (bottom panel) obtained in the emotion task, displayed separately for the six different block types and for trials with emotional and neutral faces, respectively. For RTs, main effects of block type $[F(5,65)=21.4$, $p<.001, \varepsilon=.788]$ and of valence $[F(1,13)=19.0, p<$ $.001]$ were present. RTs differed systematically between block types, being fastest in blocks including happy faces and slowest in blocks including sad faces. In addition, re- sponses were generally faster to emotional than to neutral faces. No interaction between block type and valence was obtained, indicating that this RT advantage for emotional faces was equivalent across all six block types.

For error rates, main effects of block type $[F(5,65)=$ $13.7, p<.001 ; \varepsilon=.282]$ and valence $[F(1,13)=15.4, p<$ $.002]$ were again present for the emotion task. As can be seen from Figure 2 (bottom panel), incorrect responses were most frequent in blocks including sad faces and least frequent in blocks including surprised faces. Also, it was more likely that emotional faces would be incorrectly classified as neutral than that neutral faces would be erroneously judged as emotional. No block type $\times$ valence interaction was present.

In the lines task, no main effects of block type or valence were obtained for RT or error rate (all $F \mathrm{~s}<1$ ), in-

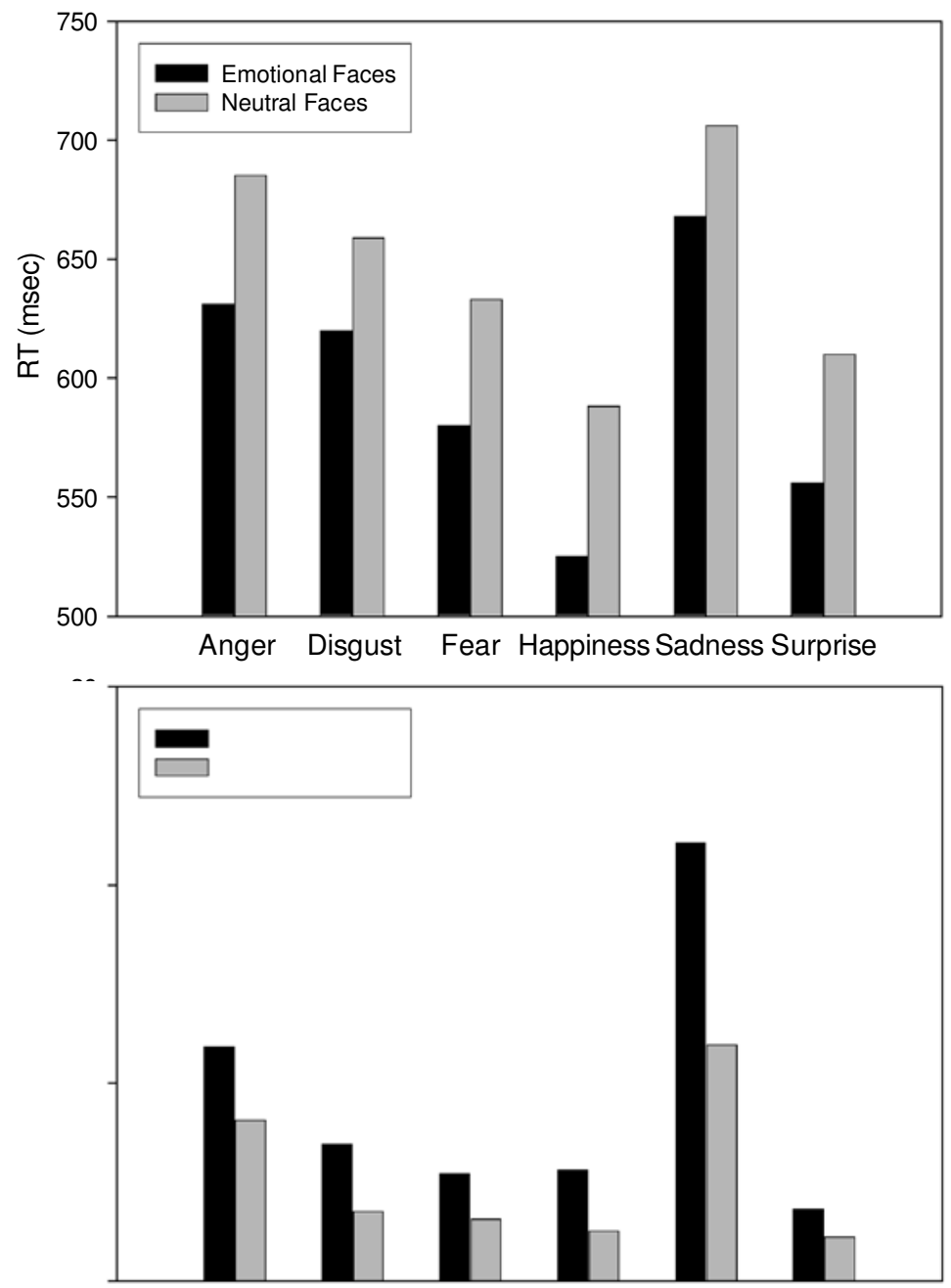

Figure 2. Reaction times (top panel) and percentage of incorrect responses (bottom panel) to emotional and neutral faces in the emotion task, displayed separately for experimental blocks where neutral faces were intermixed with angry, disgusted, fearful, happy, sad, or surprised faces. 
dicating that the emotional expression of task-irrelevant faces did not interfere with perceptual identification performance. Target type did not affect RT but had a significant effect on error rate $[F(1,13)=15.9, p<.002]$ : It was more likely that lines of different length would be classified as identical (23.2\%) than that identical lines would be judged as different (10.2\%).

\section{Electrophysiological Results}

Figure 3 shows ERPs obtained in the emotion task in response to stimulus arrays containing either neutral faces (solid lines) or emotional faces (dashed lines), collapsed across all six different emotional expressions. Figure 4 shows corresponding ERP waveforms obtained in the lines task. A sustained positivity was elicited in response to arrays containing emotional faces in the emotion task. This emotional expression effect was first visible at frontocentral sites at about $180 \mathrm{msec}$ poststimulus (overlapping with the P2 component), and appeared at parietal elec- trodes around $300 \mathrm{msec}$ poststimulus (Figure 3). At lateral temporal and occipital electrodes, emotional expression effects appeared at about $250 \mathrm{msec}$ poststimulus, as an enhanced negativity for emotional relative to neutral faces in the emotion task. In contrast, no systematic emotional expression effects were found for the lines task (Figure 4).

The difference between emotional and neutral faces appears to leave the face-specific N170 component at lateral temporal sites T5 and T6 entirely unaffected. This was observed not only in the lines task (Figure 4) but also in the emotion task (Figure 3), where facial expression was task relevant. These informal observations were substantiated by statistical analyses.

N170 component. In the N170 time range (160200 msec poststimulus), N170 amplitudes elicited at T5 and T6 in response to neutral versus emotional faces showed neither a main effect of valence nor a task $\times$ valence interaction (both $F_{\mathrm{S}}<1$ ), demonstrating that the N170 is not modulated by facial expression (Figures 3

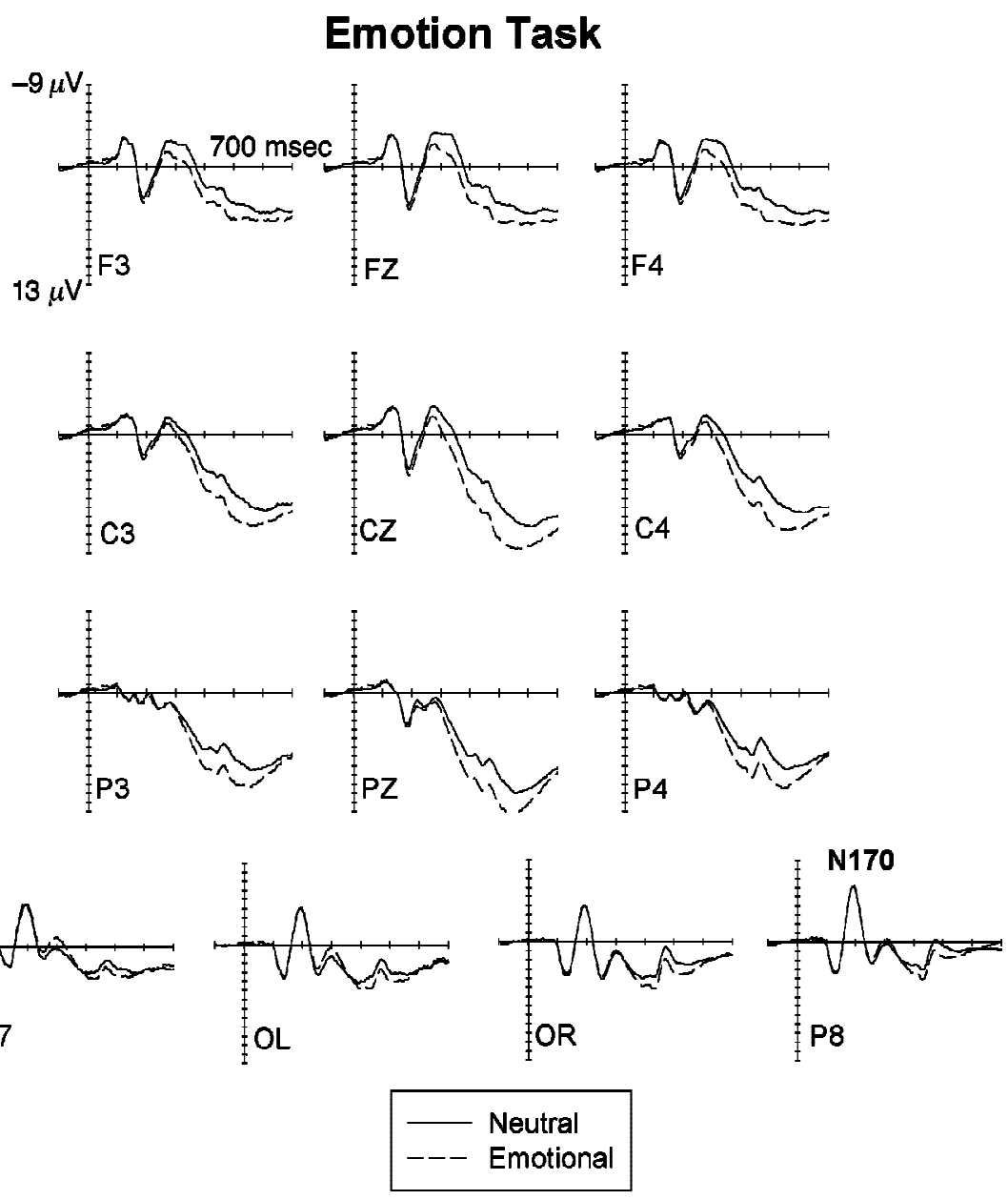

Figure 3. Grand-averaged ERP waveforms elicited in the emotion task in the 700-msec interval following stimulus onset in response to stimulus arrays containing neutral faces (solid lines) or emotional faces (dashed lines), collapsed across blocks including each of the six different emotional facial expressions. 

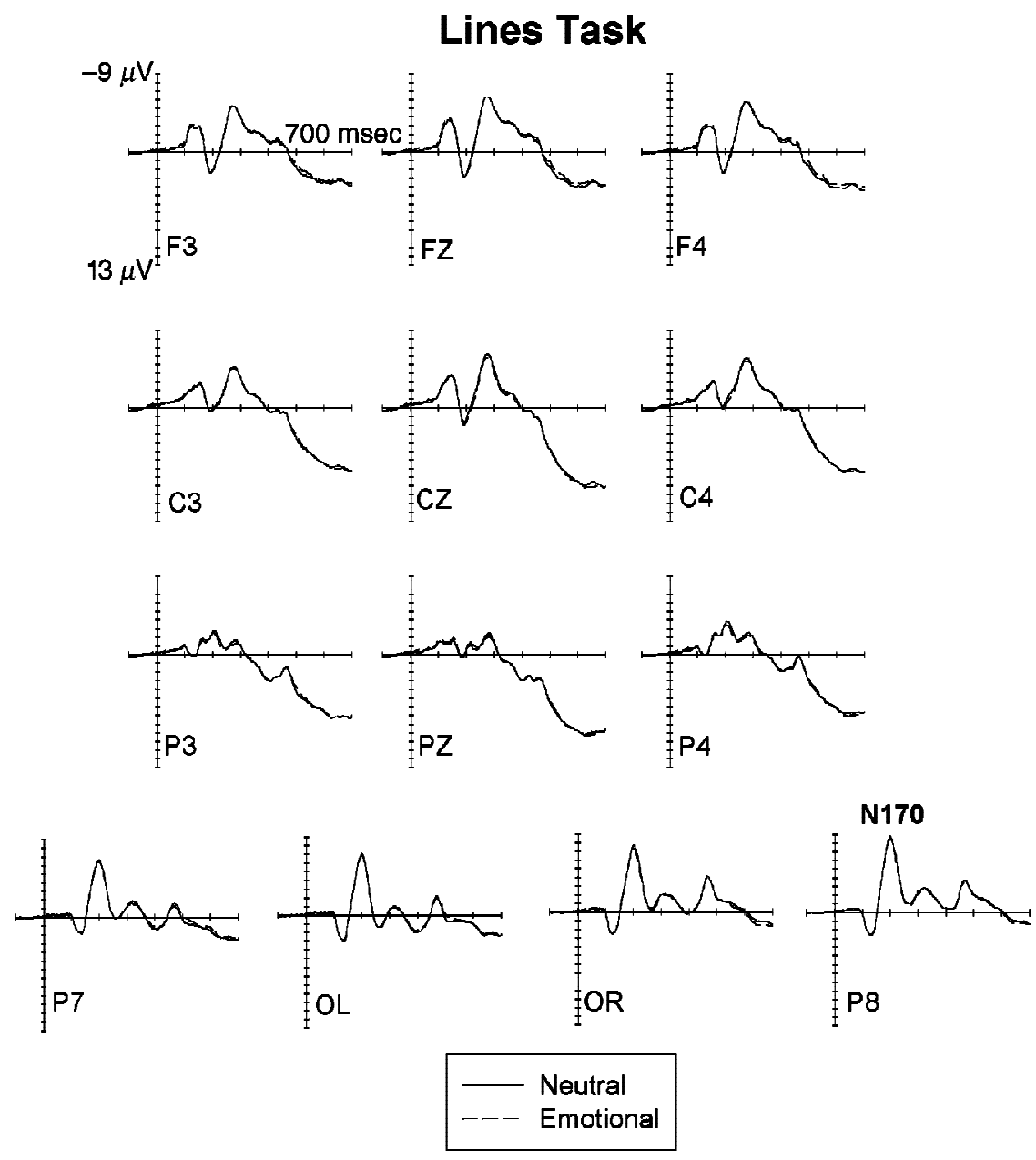

Figure 4. Grand-averaged ERP waveforms elicited in the lines task in the 700-msec interval following stimulus onset in response to stimulus arrays containing neutral faces (solid lines) or emotional faces (dashed lines). Data are collapsed across blocks including each of the six different emotional facial expressions, as well as across trials with identical and different line pairs.

and 4). To further ascertain that this component is unaffected by emotional expression even when expression is task relevant, we conducted additional analyses on N170 amplitudes observed in the emotion task (Figure 3). No main effect of valence $(F<1.2)$ or interaction between block type and valence $(F<1)$ was observed, indicating that the N170 was similarly insensitive to emotional facial expression for all six basic emotions employed here, even though participants had to discriminate between emotional and neutral faces in this task. This is illustrated in Figure 5, which displays ERPs in response to neutral and emotional faces elicited in the emotion task at right lateral temporal electrode T6, shown separately for each of the six facial expressions, which were presented in different blocks. No systematic differential effects of any facial expression on the N170 are apparent, and this was confirmed by additional planned paired comparisons of N170 amplitudes at T5 and T6 in re- sponse to emotional versus neutral faces, conducted separately for all six basic emotions. None of these comparisons even approached statistical significance [all $t \mathrm{~s}(13)<1.5]$.

Emotional expression effects. No main effects of valence or task $\times$ valence interactions were observed in the 120 - to 155 -msec time window. In the 160- to 215-msec analysis window, a task $\times$ valence interaction was present at frontal sites $[F(1,13)=5.2, p<.05] .{ }^{1}$ Main effects of valence were found at frontal and central sites [both $F_{\mathrm{s}}(1,14)>9.1$, both $\left.p \mathrm{~s}<.01\right]$ in the emotion task, reflecting an enhanced positivity elicited in response to arrays containing emotional faces (Figure 3 ). These effects were completely absent in the lines task (both $F_{\mathrm{s}}<1$ ). No interactions between block type and valence were found at frontal and central sites in the emotion task (both $F_{\mathrm{S}}<$ $1.1)$, demonstrating that this early emotional positivity was elicited in response to emotional versus neutral faces 


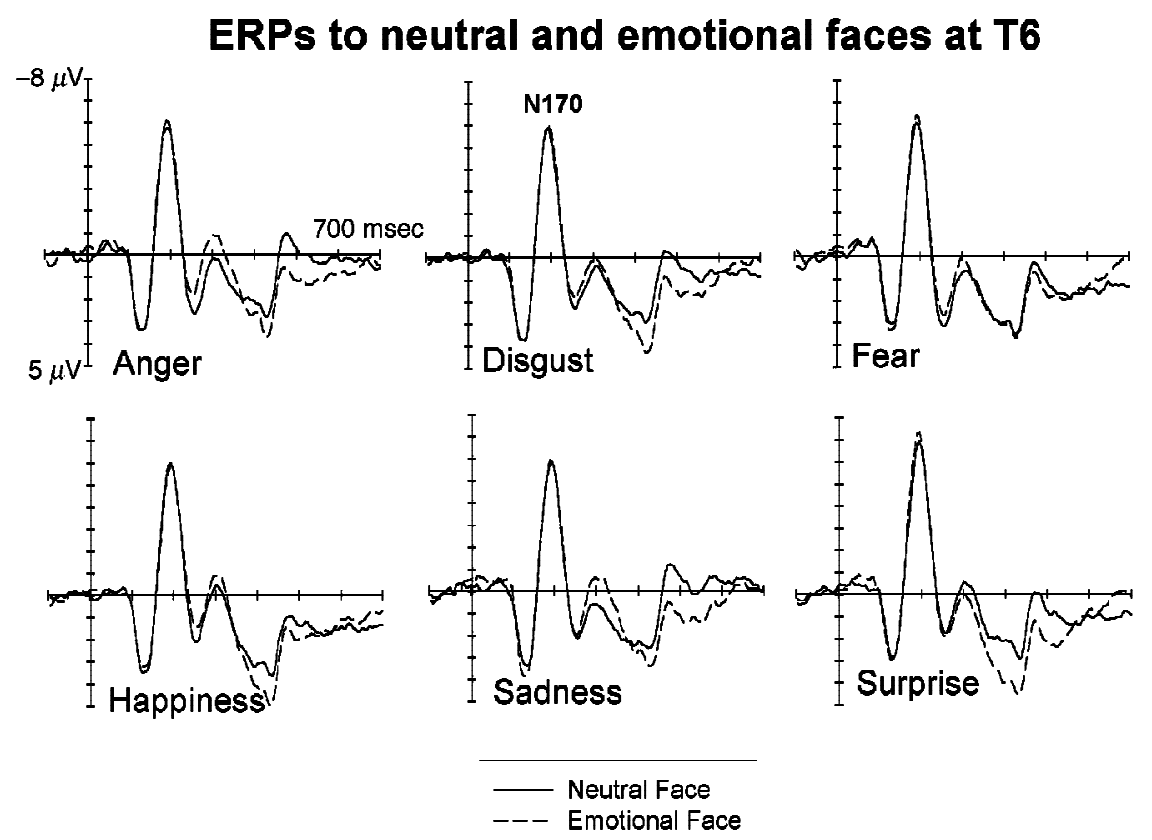

Figure 5. Grand-averaged ERP waveforms elicited in the emotion task at right lateral temporal electrode T6 in the 700-msec interval following stimulus onset in response to stimulus arrays containing neutral faces (solid lines) or emotional faces (dashed lines). ERPs are shown separately for blocks containing angry, disgusted, fearful, happy, sad, or surprised faces.

irrespective of which of the six basic emotions was included in a given block. This fact is further illustrated in Figure 6, which shows ERPs in response to neutral and emotional faces elicited in the emotion task at Fz, displayed separately for all emotional expressions used in this experiment. Emotional expression effects were very similar across expressions and started at approximately the same time for all six basic emotions. No significant emotional expression effects were present between 160 and $215 \mathrm{msec}$ poststimulus at parietal and occipital electrodes.

Between 220 and 315 msec poststimulus, task $\times$ valence interactions were present at frontal and central electrodes, as well as at lateral temporal and occipital sites [all $F_{\mathrm{s}}(1,13)>7.2$, all $p \mathrm{~s}<.02$ ], indicating that emotional expression affected ERPs in the emotion task, but not in the lines task. At frontal and central sites, main effects of valence in the emotion task [both $F_{\mathrm{s}}(1,13)>$ 15.0 , both $p$ s <.02] reflected enhanced positivities for emotional relative to neutral faces (Figure 3). No block type $\times$ valence interactions were present (both $F_{\mathrm{s}}<1$ ), demonstrating that this effect was elicited in similar fashion for all six basic emotions (Figure 6). Again, no frontocentral emotional expression effects were observed in the lines task (both $F_{\mathrm{S}}<1.6$ ). At lateral temporal and occipital sites, an enhanced negativity was observed in the 220- to 315-msec latency window for emotional relative to neutral faces in the emotion task [both $F \mathrm{~s}(1,13)>$ 6.1 , both $p \mathrm{~s}<.03$ ], but not in the lines task (both $F_{\mathrm{s}}<1$ ). Again, no block type $\times$ valence interactions were pres- ent for the emotion task (both $F_{\mathrm{S}}<1.6$ ), indicating that this lateral posterior emotional negativity was elicited in response to all six basic emotions (Figure 5).

In the final two analysis windows (320-495 msec, and 500-700 msec poststimulus, respectively), highly significant task $X$ valence interactions were present at frontal, central, and parietal electrodes [all $F_{\mathrm{S}}(1,13)>$ 10.0 , all $p \mathrm{~s}<.01$ ], again reflecting the presence of emotional expression effects in the emotion task (Figure 3) and their absence in the lines task (Figure 4). Main effects of valence at frontal and central, as well as at parietal, electrodes in the emotion task [all $F_{\mathrm{S}}(1,13)>11.9$; all $p \mathrm{~s}<.01$ ], without any significant interactions between valence and block type, demonstrated that enhanced positivities for emotional faces were elicited at these sites in a similar fashion for all six basic emotions (Figure 6). Again, effects of valence were entirely absent in the lines task. ${ }^{2}$

\section{DISCUSSION}

The primary aim of the present ERP experiment was to extend previous findings (Holmes et al., 2003) that the detection and processing of emotional information delivered by facial expressions requires focal attention. We recorded ERPs to stimulus arrays containing emotional or neutral bilateral faces under conditions when facial expression was task relevant and therefore attended (emotion task) or when attention was actively directed away from these faces toward a demanding perceptual judgment 


\title{
ERPs to neutral and emotional faces at Fz
}
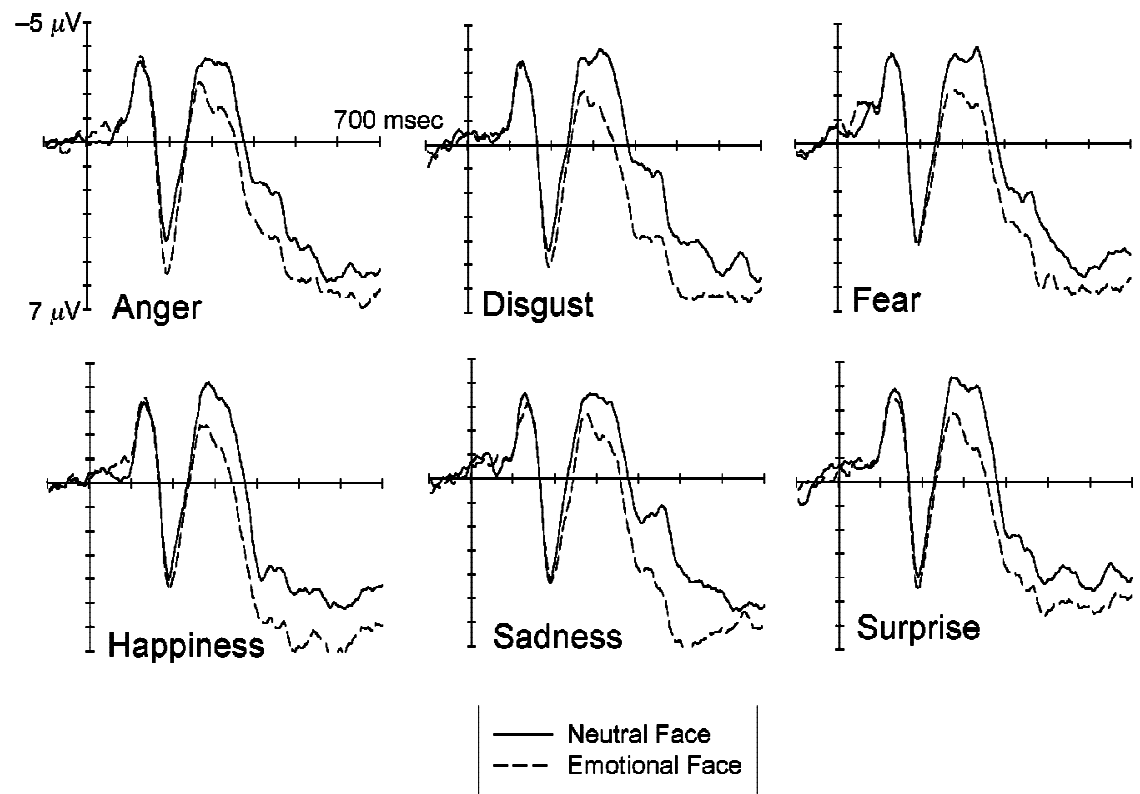

\begin{abstract}
Figure 6. Grand-averaged ERP waveforms elicited in the emotion task at midline electrode $\mathrm{Fz}$ in the 700-msec interval following stimulus onset in response to stimulus arrays containing neutral faces (solid lines) or emotional faces (dashed lines). ERPs are shown separately for blocks containing angry, disgusted, fearful, happy, sad, or surprised faces.
\end{abstract}

(lines task). In our previous ERP study (Holmes et al., 2003), spatial attention was manipulated on a trial-bytrial basis by precues presented at the start of each trial, facial expression was not task relevant (participants had to detect infrequent identical stimulus pairs, regardless of expression), and only one emotional expression (fear) was tested. In the present experiment, a sustained attention paradigm was employed (with emotion and lines tasks delivered in separate experimental halves), facial expression was task relevant in the emotion task, and, most important, all six basic facial emotional expressions were included in different blocks.

ERP correlates of emotional facial expression processing were identified by comparing ERPs elicited on trials with emotional faces with ERPs in response to neutral faces. This was done separately for the emotion task and the lines task, and for blocks including angry, disgusted, fearful, happy, sad, and surprised faces. In the emotion task, where attention was directed toward taskrelevant facial expressions, an enhanced positivity for emotional relative to neutral faces was elicited, similar to previous observations from studies comparing ERP responses to fearful versus neutral faces (Eimer \& Holmes, 2002; Holmes et al., 2003). This emotional expression effect started at about $160 \mathrm{msec}$ poststimulus and was initially distributed frontocentrally, whereas a more broadly distributed positivity was observed beyond $300 \mathrm{msec}$ (Figure 3 ). In addition, an enhanced negativity for fearful relative to neutral faces was elicited at lateral posterior electrodes between 220 and $320 \mathrm{msec}$ poststimulus.

The onset of the early frontocentral emotional expression effect was slightly later in the present experiment than in our previous experiment (Holmes et al., 2003), where significant frontal differences between ERPs to fearful and neutral faces were already present at about $120 \mathrm{msec}$ poststimulus. In the present study, vertical lines were presented close to fixation simultaneously with the bilateral faces, whereas no such stimuli were included in our earlier experiment. The presence of these additional central events may have slightly delayed the onset of early emotional expression effects. It should also be noted that an attenuation of amygdala responses to emotional facial expressions has been observed when the demand for explicit emotion recognition was increased (Critchley et al., 2000; Hariri, Bookheimer, \& Mazziotta, 2000). It is possible that the demand for explicit emotion recognition in the emotion task contributed to the delayed onset of the early emotional expression effect.

In marked contrast to these ERP results obtained in the emotion task, emotional expression effects were entirely absent in the lines task (Figure 4), demonstrating that ERP correlates of facial expression processing are strongly dependent on spatial attention. With sustained spatial attention directed away from face stimuli toward another demanding perceptual task, the presence of emotional versus neutral faces had no effect whatsoever on 
ERP waveforms. That is, emotional expression effects were completely eliminated for all six basic emotions included in this experiment. In line with this ERP result, performance in the lines task was entirely unaffected by the expression of the faces presented simultaneously with the task-relevant line pairs. Overall, these findings extend and confirm the observations of our previous ERP experiment, which compared ERPs in response to fearful versus neutral faces (Holmes et al., 2003). Clearly, these results challenge the hypothesis that the detection and/or processing of emotional facial expression occurs preattentively. If this were the case, at least some systematic ERP differences should have been elicited in response to emotional versus neutral faces in the lines task, reflecting the automatic detection of emotionally significant events.

Covert attention toward emotional faces under conditions when they were task relevant may have enhanced their visual-perceptual representation (e.g., Carrasco, Penpeci-Talgar, \& Eckstein, 2000), thereby enabling the extraction of features relating to the affective valence of these faces and thus their subsequent encoding and analysis (as reflected by the emotion-specific ERP effects observed in the emotion task). The early frontocentrally distributed emotional expression effects may be mediated by connections from the superior temporal sulcus (STS) and amygdala to orbitofrontal cortex (Rolls, 1999). The STS has been implicated in the early discrimination of visual features relating to emotional facial expressions (e.g., Sprengelmeyer et al., 1998). In addition, efferent feedback projections from the amygdala and related structures (see Lang et al., 1998; Morris et al., 1998) may have produced the more broadly distributed emotional expression effects observed in the present experiment at longer latencies.

One could argue that the absence of emotional expression effects under conditions where faces were unattended may have been due to the fact that the presentation of specific emotional expressions was blocked and that each expression was presented repeatedly in two separate blocks. Repeated exposure to a specific emotional expression may have resulted in a gradual habituation of emotion-specific responses, thus potentially attenuating any emotional expression effects that may have been present in the lines task. To investigate this possibility, we computed separate averages for the first block and for the second block including angry, disgusted, fearful, happy, sad, or surprised faces, separately for the emotion and for the lines task. These data were then analyzed with the additional factor of block position (first vs. second block containing a specific emotional facial expression). If emotional expression effects were subject to habituation, one would expect to find larger emotional expression effects for the first relative to the second block in the emotion task, and potentially also a residual emotional expression effect for the first block in the lines task.

Figure 7 shows ERPs elicited at $\mathrm{Fz}$ in response to neutral faces (solid lines) or emotional faces (dashed lines), collapsed across all six different emotional expressions. ERPs are displayed separately for the emotion task (top panel) and the lines task (bottom panel), and for the first block (left) or second block (right) including one of the six emotional expressions. As can be seen from Figure 7 (top), there was no evidence whatsoever for any habituation of emotional expression effects as a function of block position in the emotion task. This was confirmed by the absence of any block position $X$ valence or block position $\times$ block type $\times$ valence interactions for all latency windows employed in the analyses reported above [all $\left.F_{\mathrm{s}}(1,13)<1\right]$. Along similar lines, Figure 7 (bottom panel) suggests that there was no residual emotional expression effect for the first block including a specific emotional expression in the lines task. This was confirmed by the absence of any interactions involving block position [all $\left.F_{\text {s }}(1,13)<1.6\right]$. Thus, the fact that emotional expression effects were absent in response to unattended faces in the lines task is unlikely to have been the result of a habituation of emotion-specific brain responses.

The conclusion that the processing of emotional facial expression, as reflected by ERP facial expression effects, is gated by spatial attention, appears to be inconsistent with neuroimaging studies demonstrating that fearful faces result in amygdala activations even when these faces are outside the focus of attention (Vuilleumier et al., 2001; see also Morris et al., 1996; Whalen et al., 1998). However, it is extremely unlikely that the ERP effects observed in the present study are directly linked to amygdala activations. Due to its nuclear structure of clustered neurones, the amygdala is electrically closed and thus largely inaccessible to ERP measures. The early emotional expression effects observed in response to attended faces are more likely to be generated in prefrontal cortex, where emotion-specific single-cell responses have recently been recorded at short latencies (Kawasaki et al., 2001). Such prefrontal responses may reflect stages in emotional processing that could be contingent upon, but functionally separate from, prior amygdala activations (see Le Doux, 1996; Rolls, 1999). It is possible that amygdala responses can be triggered by unattended emotional stimuli (although these responses may be attenuated), whereas subsequent neocortical stages of emotional processing (as reflected by the ERP effects observed in the present experiment) are fully dependent on focal attention. An alternative possibility is that amygdala responses to emotional stimuli may also require attention (see Pessoa, Kastner, \& Ungerleider, 2002; Pessoa, McKenna, et al., 2002) and that the elimination of emotional expression effects in the lines task reflects an earlier attentional gating of such subcortical processing.

Another important new finding of the present experiment was that the onset, time course, and scalp distribution of emotional expression effects obtained in the emotion task were remarkably similar for all six basic facial expressions used here (Figures 5 and 6). The absence of any differential ERP responses to different emotional expressions was reflected by the absence of any significant 
ERPs to neutral and emotional faces at Fz EMOTION TASK

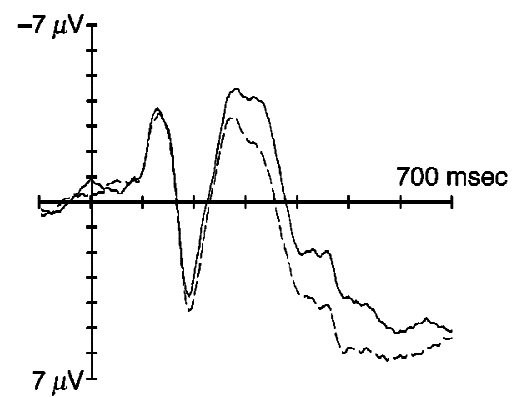

First block

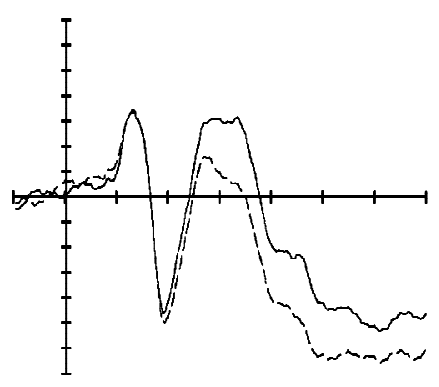

Second block

LINES TASK

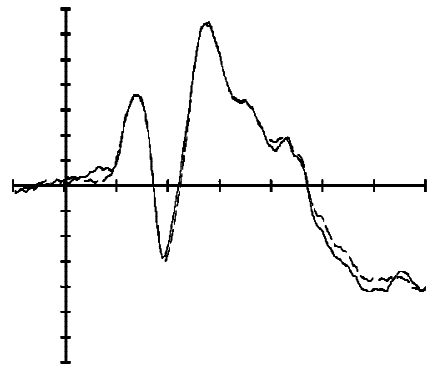

First block

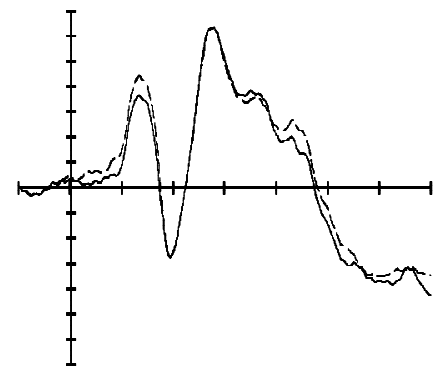

Second block

Figure 7. Grand-averaged ERP waveforms elicited in the emotion task (top panel) and in the lines task (bottom panel) at midline electrode $\mathrm{Fz}$ in the 700msec interval following stimulus onset in response to stimulus arrays containing neutral faces (solid lines) or emotional faces (dashed lines). ERPs are collapsed across blocks including each of the six different emotional facial expressions, and are shown separately for the first block (left) and the second block (right) including one specific emotional expression.

interactions between block type (blocks with angry, disgusted, fearful, happy, sad, or surprised faces) and valence (emotional vs. neutral expression). In line with these observations, the size of the RT advantage for emotional relative to neutral faces in the emotion task was similar for all six emotional facial expressions (Figure 2, top panel). The similarity in the time course of emotional expression effects across all six emotional expressions observed here suggests that emotionally relevant information delivered by facial expression is available to neocortical processes within less then $200 \mathrm{msec}$ after stimulus onset and at approximately the same time for all basic emotional expressions.

These observations do not seem to support the idea, suggested by recent fMRI results, that distinct neural subsystems specialize in the processing of specific emotions (Adolphs, 2002). If this were the case, one might have expected some systematic differences between ERP emotional expression effects elicited by different facial expressions. However, it should be noted that although some neuroimaging data show emotion-specific differential activation of brain regions such as the amygdala or insula, few studies point to differential activation within surface cortical structures (where the ERP effects observed in the present experiments are likely to be generated; see also Pizzagalli et al., 1999; Sato et al., 2001, for related results from recent ERP studies).

Thus, one could argue that early stages in the processing of emotionally relevant information, subserved by limbic structures or the basal ganglia, and subsequent neocortical emotional processing stages differ not only in their dependence on focal attention (see above) but also in their specificity. Early processes may be differentially engaged by specific emotional expressions, thus providing a rapid classification of emotionally significant events. Data in support of this view come from singleunit recordings, which reveal a rapid emergence of differential effects to emotional expressions in the human 
amygdala (Liu et al., 1999). Conversely, later stages might be involved in the in-depth processing of various kinds of affective information and thus would be much less selective with respect to different facial expressions.

This suggestion is consistent with some recent evidence that subcortical and neocortical routes for visual processing are involved differentially in emotional expression analysis. A subcortical magnocellular pathway to the amygdala would appear to support valence discrimination processes, whereas parvocellular subsystems of ventral visual cortices may be preferentially involved in emotional intensity evaluation, irrespective of emotional valence (Schyns \& Oliva, 1999; Vuilleumier, Armony, Driver, \& Dolan, 2003). Recent neuroimaging results (Vuilleumier et al., 2003) suggest that low and high spatial frequency components of fearful faces selectively drive amygdala and visual cortical responses, respectively. However, although enhanced amygdala activation was found in response to low-spatial-frequency fearful face stimuli, explicit judgments relating to the perceived intensity of fearfulness were increased by the presence of high-spatial-frequency cues. These results support the view that coarse visual information may be directed via magnocellular channels from the retina to the amygdala through a tectopulvinarpathway (e.g., Bisti \& Sireteanu, 1976; Jones \& Burton, 1976), enabling the fast appraisal of the affective significance of a stimulus (e.g., Morris, Öhman, \& Dolan, 1999). ${ }^{3}$

Another aim of the present study was to investigate whether the face-specific N170 component, which is assumed to reflect the structural encoding of faces, is sensitive to emotional facial expressions. In previous ERP studies, which have not found any modulations of the N170 elicited by fearful relative to neutral faces (Eimer \& Holmes, 2002; Holmes et al., 2003), facial expression was always task irrelevant. In contrast, participants' responses were contingent upon facial expression in the present emotion task. In spite of this fact, the N170 was found to be completely unaffected by facial expressions in the emotion task, and this was consistently the case for all six emotional expressions used in the present study (Figure 5).

In line with earlier findings from depth electrodes (McCarthy, Puce, Belger, \& Allison, 1999), this pattern of results now demonstrates comprehensively that the structural encoding of faces, as reflected by the N170, is entirely insensitive to information derived from emotional facial expression. Thus, the rapid detection of emotional facial expression appears to occur independently and in parallel to the construction of a detailed perceptual representation of a face. The absence of systematic early emotional expression effects at posterior sites, and the presence of such ERP effects at frontocentral electrodes at about $160 \mathrm{msec}$ poststimulus, suggests that higher order visual processing stages involved in face processing are affected by emotional facial expression only after this information has been processed in prefrontal cortex. This is consistent with the face processing model proposed by Bruce and Young (1986), in which the extraction of per- ceptual information for emotional expression processing occurs independently and simultaneously with structural encoding for face recognition.

In summary, the present ERP results demonstrate that the neocortical processing of emotional facial expression is strongly dependent on focal attention. When faces were attended, systematic emotional expression effects were elicited by emotional relative to neutral faces, and these effects were strikingly similar in terms of their timing and morphology for all six basic facial expressions. In contrast, when attention was actively directed away from these faces, emotional expression effects were completely eliminated. The rapid and automatic encoding of emotionally significant events occurring outside the focus of attention may be adaptively advantageous, because it prepares the organism for fight or flight through subcortically mediated autonomic activation (e.g., Öhman, Flykt, \& Lundqvist, 2000). However, it is equally important that irrelevant affective stimuli do not continuously divert attention. This suggests a division of labor between limbic structures involved in the obligatory detection of emotional information-preparing the organism for rapid action (Morris et al., 1999; Whalen et al., 1998) — and subsequent neocortical emotional processing stages. Limbic structures may be responsible for establishing a readiness to respond to any environmental threat that could become the focus of attention, presumably through heightened autonomic activation. However, neocortical stages appear to be protected by efficient attentional gating mechanisms, which reduce distractibility by emotional stimuli so that ongoing goals and plans can be accomplished without interference from irrelevant events.

\section{REFERENCES}

Adolphs, R. (2002). Recognizing emotion from facial expressions: Psychological and neurological mechanisms. Behavioral Cognitive Neuroscience Review, 1, 21-61.

Adolphs, R., Tranel, D., \& Damasio, A. R. ( 2003). Dissociable neural systems for recognizing emotions. Brain \& Cognition, 52, 6169.

Amaral, D. G., \& Price, J. L. (1984). Amygdalo-cortical projections in the monkey (Macaca fasicularis). Journal of Comparative Neurology, 230, 465-496.

Amaral, D. G., Price, J. L., Pitkanen, A., \& Carmichael, S. T. (1992). Anatomical organization of the primate amygdaloid complex. In J. P. Aggleton (Ed.), The amygdala: Neurobiological aspects of emotion, memory, and mental dysfunction (pp. 1-66). New York: Wiley-Liss.

Armony, J. L., \& Dolan, R. J. (2002). Modulation of spatial attention by fear-conditioned stimuli: An event-related fMRI study. Neuropsychologia, 7, 817-826.

Bentin, S., Allison, T., Puce, A., Perez, E., \& McCarthy, G. (1996). Electrophysiological studies of face perception in humans. Journal of Cognitive Neuroscience, $\mathbf{8}, 551-565$.

Bisti, S., \& Sireteanu, R. C. (1976). Sensitivity to spatial frequency and contrast of visual cells in the cat superior colliculus. Vision Research, 16, 247-251.

Blair, R. J. R., Morris, J. S., Frith, C. D., Perrett, D. I., \& Dolan, R. J. (1999). Dissociable neural responses to facial expressions of sadness and anger. Brain, 122, 883-893.

Breiter, H. C., Etcoff, N. L., Whalen, P. J., Kennedy, W. A., Rauch, S. L., Buckner, R. L., Strauss, M. M., Hyman, S. E., \& 
Rosen, B. R. (1996). Response and habituation of the human amygdala during visual processing of facial expression. Neuron, 17, 875887.

Bruce, V., \& Young, A. (1986). Understanding face recognition. British Journal of Psychology, 77, 305-327.

Calder, A. J., Keane, J., Manes, F., Antoun, N., \& Young, A. W. (2000). Impaired recognition and experience of disgust following brain injury. Nature Neuroscience, 3, 1077-1078.

Calder, A. J., Lawrence, A. D., \& Young, A. W. (2001). Neuropsychology of fear and loathing. Nature Reviews Neuroscience, 2, 352363.

Carrasco, M., Penpeci-Talgar, C., \& Eckstein, M. (2000). Spatial covert attention increases contrast sensitivity across the CSF: Support for signal enhancement. Vision Research, 40, 1203-1215.

Critchley, H. [D.], Daly, E. [M.], Phillips, M., Brammer, M., Bullmore, E., Williams, S. [C.], van Amelsvoort, T., Robertson, D., DAVID, A., \& MurPHY, D. [G. M.] (2000). Explicit and implicit neural mechanisms for processing of social information from facial expressions: A functional magnetic resonance imaging study. Human Brain Mapping, 9, 93-105.

Cuthbert, B. N., Schupp, H. T., Bradley, M. M., Birbaumer, N., \& LANG, P. J. (2000). Brain potentials in affective picture processing: Covariation with autonomic arousal and affective report. Biological Psychology, 52, 95-111.

DAmASIO, A. R. (1994). Descartes' error: Emotion, reason, and the human brain. New York: G. P. Putnam's Sons.

Diedrich, O., Naumann, E., Maier, S., \& Becker, G. (1997). A frontal slow wave in the ERP associated with emotional slides. Journal of Psychophysiology, 11, 71-84.

Eastwood, J. D., Smilek, D., \& Merikle, P. M. (2001). Differential attentional guidance by unattended faces expressing positive and negative emotion. Perception \& Psychophysics, 63, 1004-1013.

EIMER, M. (1998). Does the face-specif ic N170 component reflect the activity of a specialized eye detector? NeuroReport, 9, 2945-2948.

Eimer, M. (2000). The face-specific N170 component reflects late stages in the structural encoding of faces. NeuroReport, 11, 2319-2324.

Eimer, M., \& Holmes, A. (2002). An ERP study on the time course of emotional face processing. NeuroReport, 13, 427-431.

Ekman, P., \& Friesen, W. V. (1976). Pictures offacial affect. Palo Alto, CA: Consulting Psychologists Press.

Fox, E., Lester, V., Russo, R, Bowles, R. J., Pichler, A, \& Dutton, K. (2000). Facial expressions of emotion: Are angry faces detected more efficiently? Cognition \& Emotion, 14, 61-92.

Hansen, C. H., \& Hansen, R. D. (1988). Finding the face in the crowd: An anger superiority effect. Journal of Personality \& Social Psychology, 54, 917-924.

Hariri, A. R. Bookheimer, S. Y., \& Mazziotta, J. C. (2000). Modulating emotional responses: Effects of a neocortical network on the limbic system. NeuroReport, 11, 43-48.

Harmer, C. J., Thilo, K. V., Rothwell, J. C., \& Goodwin, G. M. (2001). Transcranial magnetic stimulation of medial-frontal cortex impairs the processing of angry facial expressions. Nature Neuroscience, 4, 17-18.

Holmes, A., Vuilleumier, P., \& Eimer, M. (2003). The processing of emotional facial expression is gated by spatial attention: Evidence from event-related brain potentials. Cognitive Brain Research, 16, 174-184.

Jones, E. G., \& Burton, H. (1976). A projection from the medial pulvinar to the amygdala in primates. Brain Research, 104, 142-147.

Kawasaki, H., Kaufman, O., Damasio, H., Damasio, A. R, Granner, M., Bakken, H., Hori, T., Howard, M. A., III, \& Adolphs, R. (2001). Single-neuron responses to emotional visual stimuli recorded in human ventral prefrontal cortex. Nature Neuroscience, 4, 15-16.

Lane, R. D., Chua, P. M., \& Dolan, R. J. (1999). Common effects of emotional valence, arousal and attention on neural activation during visual processing of pictures. Neuropsychologia, 37, 989-997.

Lang, P. J., Bradley, M. M., Fitzsimmons, J. R., Cuthbert, B. N., Scott, J. D., Moulder, B., \& NAngia, V. (1998). Emotional arousal and activation of the visual cortex: An fMRI analysis. Psychophysiology, 35, 199-210.
LE Doux, J. E. (1996). The emotional brain. New York: Simon \& Schuster.

LiU, L., IoAnnides, A. A., \& Streit, M. (1999). Single trial analysis of neurophysiological correlates of the recognition of complex objects and facial expressions of emotion. Brain Topography, 11, 291-303.

McCarthy, G., Puce, A., Belger, A., \& Allison, T. (1999). Electrophysiological studies of human face perception II. Response properties of face-specific potentials generated in occipitotemporal cortex. Cerebral Cortex, 9, 431-444.

MogG, K., \& Bradley, B. P. (1999). Orienting of attention to threatening facial expressions presented under conditions of restricted awareness. Cognition \& Emotion, 13, 713-740.

Mogg, K., McNamara, J., Powys, M., Rawlinson, H., Seiffer, A., \& Bradley, B. P. (2000). Selective attention to threat: A test of two cognitive models of anxiety. Cognition \& Emotion, 14, 375-399.

Morris, J. S., Friston, K. J., Buechel, C., Frith, C. D., Young, A. W., Calder, A. J., \& Dolan, R. J. (1998). A neuromodulatory role for the human amygdala in processing emotional facial expressions. Brain, 121, 47-57.

Morris, J. S., Frith, C. D., Perrett, D. I., Rowland, D., Young, A. W., Calder, A. J., \& Dolan, R. J. (1996). A differential neural response in the human amygdala to fearful and happy facial expressions. Nature, $\mathbf{3 8 3}, 812-815$.

Morris, J. S., Öhman, A., \& Dolan, R. J. (1999). A subcortical pathway to the right amygdala mediating "unseen" fear. Proceedings of the National Academy of Sciences, 96, 1680-1685.

Öhman, A., Fly Kт, A., \& Esteves, F. (2001). Emotion drives attention: Detecting the snake in the grass. Journal of Experimental Psychology: General, 130, 466-478.

Öhman, A., Fly Kt, A., \& Lundqvist, D. (2000). Unconscious emotion: Evolutionary perspectives, psychophysiological data and neuropsychological mechanisms. In R. D. Lane \& L. Nadel (Eds.), Cognitive neuroscience of emotion (pp. 296-327). New York: Oxford University Press.

Öhman, A., Lundqvist, D., \& Esteves, F. (2001). The face in the crowd revisited: A threat advantage with schematic stimuli. Journal of Personality \& Social Psychology, 80, 381-396.

Pessoa, L., Kastner, S., \& Ungerleider, L.G. (2002). Attentional control of the processing of neutral and emotional stimuli. Cognitive Brain Research, 15, 31-45.

Pessoa, L., McKenna, M., Gutierrez, E., \& Ungerleider, L.G. (2002). Neural processing of emotional faces requires attention. Proceedings of the National Academy of Sciences, 99, 11458-11463.

Phillips, M. L., Young, A. W., Scott, S. K., Calder, A. J., Andrew, C., Giampietro, V., Williams, S. C. R, Bullmore, E. T., Brammer, M., \& GRAY, J. A. (1998). Neural responses to facial and vocal expressions of fear and disgust. Proceedings of the Royal Society London: Series B, 265, 1809-1817.

Phillips, M. L., Young, A. W., Senior, C., Brammer, M., Andrew, C., Calder, A. J., Bullmore, E. T., Perrett, D. I., Rowland, D., \& Williams, S. C. R, ET AL. (1997). A specific neural substrate for perceiving facial expressions of disgust. Nature, 389, 495-498.

Pizzagalli, D., Regard, M., \& Lehmann, D. (1999). Rapid emotional face processing in the human right and left brain hemispheres: An ERP study. NeuroReport, 10, 2691-2698.

Rapcsak, S. Z, Galper, S. R., Comer, J. F., Reminger, S. L., Nielsen, L., Kasziniak, A. W., Verfaellie, M., Laguna, J. F., Labiner, D. M., \& Cohen, R. A. (2000). Fear recognition deficits after focal brain damage. Neurology, 54, 575-581.

RoLls, E. T. (1999). The brain and emotion. Oxford: Oxford University Press.

Sato, W., Kochiyama, T., Yoshikawa, S., \& Matsumura, M. (2001). Emotional expression boosts early visual processing of the face: ERP recording and its decomposition by independent component analysis. NeuroReport, 12, 709-714.

Schyns, P. G., \& Oliva, A. (1999). Dr. Angry and Mr. Smile: When categorization flexibly modifies the perception of faces in rapid visual presentations. Cognition, 69, 243-265.

Sprengelmeyer, R, Rausch, M., Eysel, U. T., \& Przuntek, H. (1998). Neural structures associated with recognition of facial expressions of 
basic emotions. Proceedings of the Royal Society London: Series B, 265, 1927-1931.

STEPNIEWSKA, I., QI, H. X., \& KAAS, J. H. (1999). Do superior colliculus projection zones in the inferior pulvinar project to MT in primates? European Journal of Neuroscience, 11, 469-480.

Vuilleumier, P., Armony, J. L., Driver, J., \& Dolan, R. J. (2001). Effects of attention and emotion on face processing in the human brain: An event-related fMRI study. Neuron, 30, 829-841.

Vuilleumier, P., Armony, J. L., Driver, J., \& Dolan, R. J. (2003). Distinct spatial frequency sensitivities for processing faces and emotional expressions. Nature Neuroscience, 6, 624-631.

Vuilleumier, P., \& Schwartz, S. (2001a). Beware and be aware: Capture of spatial attention by fear-related stimuli in neglect. NeuroReport, 12, 1119-1122.

Vuilleumier, P., \& Schwartz, S. (2001b). Emotional expressions capture attention. Neurology, 56, 153-158.

Whalen, P. J., Rach, S. L., Etcoff, N. L., McInerney, S. C., Lee, M. B., \& JENIKE, M. A. (1998). Masked presentations of emotional facial expressions modulate amygdala activity without explicit knowledge. Journal of Neuroscience, 18, 411-418.

Whalen, P. J., Shin, L. M., McInerney, S. C., Fischer, H., Wright, C. I., \& RAUCH, S. L. (2001). A functional MRI study of human amygdala responses to facial expressions of fear versus anger. Emotion, 1, 70-83.

Yeterian, E. H., \& Pandya, D. N. (1991). Corticothalamic connections of the superior temporal sulcus in rhesus monkeys. Experimental Brain Research, 83, 268-284.

\section{NOTES}

1. In spite of the fact that significant valence effects were present at central electrodes in the emotion task, but were absent in the lines task, this interaction failed to reach significance at central sites.

2. At lateral occipital electrodes, a significantly enhanced positivity for emotional relative to neutral faces was present between 320 and $495 \mathrm{msec}$ in the emotion task $[F(1,13)=6.2, p<.03]$, but not in the lines task, and this was reflected in a nearly significant task $\times$ valence interaction $[F(1,13)=4.6, p<.06]$.

3. It should be noted that anatomical evidence for a colliculo-pulvinaramygdalar pathway is currently lacking, since the medial pulvinar, which projects to the amygdala, does not receive a significant direct input from the superior colliculus (e.g., Stepniewska, Qi, \& Kaas, 1999). However, possible connections between the inferior pulvinar (which receives visual inputs from the superior colliculus) and the medial nucleus may support the transmission of information to the amygdala through a colliculo-pulvinar route. Alternatively, cortical input may be involved, since STS (implicated in facial expression processing; Sprengelmeyer et al., 1998) is known to project to the medial pulvinar (Yeterian \& Pandya, 1991). Our thanks to an anonymous reviewer for raising this important point.

(Manuscript received December 10, 2002; revision accepted for publication June 11,2003 .) 